\title{
Exploration on Influence of Development of High-tech Architecture on Contemporary Chinese Architecture
}

\author{
Minghui Li, Shijie Zhang, Xu Guo
}

\author{
Qilu University of Technology, Jinan Shandong, 250353, China
}

Key words: High-tech, Architecture, Technology, Design.

\begin{abstract}
Originating from 1960s, high-tech architecture has developed into one of the important schools in the circle of contemporary architecture. This paper mainly describes development technologies and future development trend of Chinese contemporary architecture under the inspiration of high-tech school through analysis on the development of high-tech architecture, puts forward influence and promotion points of high-tech school for Chinese contemporary architecture based on the status quo of Chinese architecture, forms feasible innovation and integration points and provides referential thinking and development direction for Chinese building industry and architectural designers.
\end{abstract}

\section{Introduction}

High-tech architecture has emerged and become an extremely active architecture school in 1960s when the trend of diversification of world architectural art began. Since ancient times, the development of architectural history has always conformed to a certain law, i.e. new demands for architecture often accompany great change of the society, which urges people to make constant explorations, find technical means meeting such demands finally, e.g. new materials, new structure and new process, solve the problem and adapt to the requirement of social development. New materials, process and structure have been promoted and applied and constantly improved. Therefore, in-depth discussion and study on it have great significance for the development of Chinese architecture cause.

\section{High-tech architecture}

High-tech school pays attention to the use of contemporary high-tech achievements in architecture to show the beauty of architectural art, expands understandings and expression of architecture constantly and create buildings respecting historic culture and spirit of the age.

\section{Connotations and concept of high-tech school}

High-tech school is explained as below in the textbook of architectural history: "the tendency of paying attention to high industrial technology refers to the tendency of adhering to adopt new technologies in architecture and meanwhile advocating presenting new technologies in aesthetics". High-tech school is an architectural school using advanced science and technology to reflect aesthetic value of architectural technology. From the perspective of aesthetics, architects of the school show their advocation of technical aesthetics through structure, materials and equipment of modern architecture and convey their praise of modern science and technology civilization.

In terms of concept, high-tech school advocates aesthetics of new technology, emphasizes the importance of mechanical equipment, structure and pipeline and attempts to make architecture show the effect of separation from tradition and environment and discontinuous jumping. Modern steel structure buildings represented by high-tech architecture mostly present the beauty of technology with the shape of steel framework and exposure of structural members. Most buildings give a feeling of tension and strength with the tension of diagonal member. 


\section{Development and restriction of high-tech school}

In 1950s, high-tech architecture mainly focused on the adoption of high and new technologies and the assembly and standardization of components. In 1960s, it was characterized by emphasis on and manifestation of modern industrial technology. Since 1970s, restrictions of high-tech school have been increasingly prominent. Early high-tech architecture ignored fine sentiments of human beings and had obvious deficiencies in energy conservation, environmental protection and coordination with urban environment. With increasing deterioration of ecological environment and the outbreak of global energy crisis, high-tech school characterized by the presentation of modern industrial technology has started to pay attention to local culture, regional climate and ecological environment. The most powerful content in the thought of high-tech architecture is the attention to ecological environment. This is just the specialty of high-tech school. Meanwhile, globalization tendency of culture has triggered the importance attached to regional culture and historical consciousness. High-tech school has constantly created many new works and expressed design concept with brand new scientific and technological means.

\section{Inevitable trend of high-tech architecture in current social development}

With the coming of information society, high-tech architects have changed their architectural orientation to adapt to perfect unification of high technology in the information era with natural environment, urban context, ecological protection, energy utilization and demands of human and realize the environmental concept of green, science and technology and adaptation to sustainable suitability for residence. Such change has formed the inevitable trend of high-tech architecture in current social development in terms of urban environment, historic context, ecological environmental protection and artistic feeling.

\section{Integrate with urban environment and become city card. Placeness - from performance in city to existence for city.}

Space concept of early high-tech architects is an extreme isotropic space. Such space concept does not simply remains indoors. When outdoor environment is processed, architecture is still superior to environment and a unique and fantastic landscape is created in city with its strong self-expression ability. Therefore, its work is often not integrated with the environment and even contradictory to the environment.

Gary Art Center in Lesey City, a famous historic and cultural city in France, was built on the ruins of the $19^{\text {th }}$ century opera house near Mason Lesey Temple in Rome. In such profound atmosphere of traditional culture, Forster integrated it into the historic street with his refined high-tech design concept. It respected history, resonated with the temple and fully expressed the respect of architect to the site. Therefore, contemporary high-tech architects should not only use local materials to simulate details and symbols of surrounding buildings traditionally, but also use new materials to obtain a "counterpoint" coordinating relation with surrounding buildings in urban scale, space particularity and detail processing with their superb skills and sensitive and unique understandings of city.

\section{Integrate with historic context and reflect heritage. Contextuality - from objection to historic contextualism to importance attached to historic and regional context.}

High-tech architecture originally objected to historic contextualism. It has created shocking artistic images with "single decoding" of high technology but disdained association with any history. However, the current trend of aesthetic softening makes the integration of such confrontation inevitable as attaching importance to the continuation of architectural historic context does not mean abandoning pursuit for high technology; instead, it requires people to experience the influence of cultural and historic environment on people living therein, thus making the life of people richer and meeting the demand of people for high sentiment in the current society. Tjibaou Cultural Center designed by Renzo Piano was built with modern technology under the inspiration of traditional form and successfully memorized and explained Melanesian culture of Karox. 


\section{Integrate with ecological environmental protection and realize energy-saving and green development. Ecological feature - ecological high-tech architecture from "artificial" to "natural" feature.}

Early high-tech architects were addicted to unprecedented large span, flexible space and new equipment but sacrificed natural lighting and ventilation. Architects from various countries in the world integrate the concept of "ecology and environmental protection" and "sustainable development" into architectural design spontaneously. Architectural technology starts to be "green". For example, the application of solar reflective materials, optically controlled sunshade components and various new outer walls controlling solar radiation and heat entry not only saves energy effectively, but also adds the charm of architecture appearance and gives people a strong sense of "science and technology".

It should be noted that such "high technology" does not make a "green" architecture simply with artificial equipment and various new materials; instead, traditional design concept is changed through "technical thinking" and a new energy-saving system is created with the principle of ecology and the design concept of high information, low energy consumption, recyclability and self-adjusting ability.High-tech architects of the new generation are committed to creating an ideal living environment with new achievements in modern process technology and meanwhile protecting ecological environment and reducing energy consumption and pollution to the greatest extent, i.e. taking a path of sustainable development and forming corresponding path and features of high-tech ecological architecture, thus developing its core role in the development process of high-tech school.

\section{Integrate with artistic feeling and build the feeling of beauty. Artistry - from "high technology" to "high-tech art".}

Technology is rooted in the past, controls today and looks into the future. When technology completes its real mission, it sublimates to architectural art. Technical aesthetics of early high-tech school was adapted to "industrial age", advocated high efficiency, conciseness and unusual large scale of machine, had closer relation with the rationality of function, production process and construction, tended to logical thinking and considered process, operation, technical facilities and structure, but had negative attitude towards art. The new generation of high-tech architecture has gradually become creative art. It no longer denies art. Instead, it combines structure and art organically, stimulates mental field of people with flexible, exaggerated and diversified concepts and makes structure and technology an elegant "high-tech art". Forster said when interviewed by the journalist of French Architectural Journal, "some people praise our building as the most touching and refined one in modern architecture so far. I believe that these buildings reflect deep concern about quality. Quality has become ideology and technology becomes creative art $\cdots . "$

\section{Analysis on Chinese high-tech architecture and development suggestions}

Under the influence of design concept of sustainable development of modern information society, Chinese contemporary architecture pays more attention to architecture and natural environment, urban context, ecological environmental protection and emotional needs of human beings. The design of many exhibition halls in Shanghai World Expo shows highlights of Chinese high-tech architecture. For example, in circular arc Saudi Arabia pavilion supported in the air which was designed by Chinese company, dates were planted on the ground and roof just like an emerald as well as a "treasure ship" slipping through the waves, forming a hanging garden with shadows of trees dancing in the breeze and dense desert style. Lighting and ventilation of the pavilion fully consider regional features of Shanghai. The dominant ramp design provides convenience for the disabled and the blind. It can be called as a people-oriented environmentally friendly building. The implied meaning of "silk road treasure ship" is that Maritime Silk Road connects Arabian and Chinese civilization. Therefore, new technology shown by Chinese high-tech architecture in Shanghai World Expo is manifested in not only the use of latest materials and technical means, but also the attention paid to new technical ideology and frontier issues such as ecology, energy conservation and sustainable development. 
High-tech architecture conveys cultural information through specific formal language with technical means as medium, locates design creativity from historic and cultural perspectives and makes high-tech architecture personal and no longer as cold as machine aesthetic architecture.

Second, Chinese high-tech architecture forms rich formal language based on machine aesthetic architecture and improves architectural function with technical means. It inherits experimental idea of modernist art and the tendency of beauty of architectural technology and meanwhile promotes the beauty of architectural technology towards diversification and avoids the limitation of machine aesthetic theory and oneness of architectural form. Rich architectural art is expressed with high technology. While paying attention to technology, it does not reject culture and history. Moreover, highly sensitive system is installed for architecture by means of computer control technology. Architecture is controlled uniformly by computer through sensor, thus becoming an interactive organic integrity.

The future new century is an era paying attention to ecology and environmental protection and pursuing for overall coordinated development of human and natural science, which requires the support of green design concept and the integration of more humanistic care to form high technology and sentiment in humanistic environment. Architectural design has dual characters of physical and spiritual functions. Design should more importantly meet the requirement of spiritual function on the basis of meeting physical function and create style, artistic conception and sentiment to meet aesthetic requirements of people while paying attention to high technology. Specific manifestations are concise image, kind modeling, transparent economy, diversified functions, natural, toxic-free and reproducible materials and careful detail design. An ideal residential environment should be created. Future architectural design will be the combination of technology and ecology under the guidance of high-tech aesthetics and ecological aesthetics.

China has resources, ability and foundation and can contest with all kinds of international buildings as China has five thousand years of cultural deposits. China is showing the application of modern scientific and technological revolution with its rapid development mode. The introduction of western design thought gives a guiding light to Chinese designers and helps and encourages the prosperous development of Chinese architecture.

\section{Conclusion}

In the current era with different schools and co-existence of diversified cultures, how to create characteristic works with rapport in the context filled with sense of history is still worth pondering by local architects. Faced with the trend, architects in the new era should absorb the quintessence from various traditional and modern style schools. On the one hand, they should maintain their original optimism and confidence in technology and continue to inject new materials, technologies and thinking into architectural works. On the other hand, they should also pay attention to city, environment, energy conservation, environmental protection, nature and human feelings and develop their high-tech advantages to emotional field boldly so as to create buildings with spirit of the age and high sentiment respecting history and culture. Only in this way can high-tech architecture get rid of early restrictions and step into a diversified emotional world with humanistic care.

Today, high-tech architecture is rising and in the ascendant in China. Architectural language is the medium of exchange among people and among cultures. New vital force can be obtained by attaching importance to technical science education, improve the level of professional talents and professional accomplishment of designers, use multi-disciplinary thought for reference, establish appropriate technology view correctly, paying attention to cultural psychology structure formed under specific cultural background, expanding connotations of technical functions, covering the scope of social mentality, fully undertaking responsibilities endowed by the era and the society and making architecture actually become the architecture of "human". Only high technology rooted in this is the real high technology in the $21^{\text {st }}$ century. Only homeland beloved by people will become the homeland in the real sense. 


\section{References}

[1] Tongji University, Tsinghua University, Nanjing Institute of Technology etc. A History of Modern Architecture in the Foreign Countries, Beijing: China Building Industry Press, 1998.

[2] Jiang Wei. Discussion on Tendency of Emotionalization of Contemporary High-tech Architecture, Architect, 2001. 40-42.

[3] Zeng Qun. Relativity and Eternity of High-tech Architecture, Architectural Forum, 2003 (2): 56-58.

[4] Lin Qing. Collection of Excellent Works of World Master Architects: Norman Forster, Beijing: China Building Industry Press, 1999. 